\title{
VISCOSITY LIMIT OF STATIONARY DISTRIBUTIONS FOR THE RANDOM FORCED BURGERS EQUATION
}

\author{
DIOGO GOMES, RENATO ITURRIAGA, KONSTANTIN KHANIN PABLO \\ PADILLA
}

\begin{abstract}
We prove convergence of stationary distributions for the randomly forced Burgers and Hamilton-Jacobi equations in a limit when viscosity tends to zero. It turns out that for all values of the viscosity $\nu$ there exists a unique (up to an additive constant) solution to the randomly forced Hamilton-Jacobi equation which is extendible for all times. The main result follow from the convergence of these solutions in a limit when $\nu$ tends to zero without changing its sign. The two limiting solutions (for different signs of the viscosity term) correspond to unique backward and forward viscosity solutions. Our approach, which is an extension of the previous work [6], [14], is based on the stochastic version of Lax formula for solutions to the initial and final value problems for the viscous Hamilton-Jacobi equation.
\end{abstract}

\section{INTRODUCTION}

In this paper we study the viscosity limit for Burgers equation:

$$
u_{t}+(u \cdot \nabla) u=\nu \Delta u+f(x, t)
$$

where $u(x, t)=\left(u_{i}(x, t), 1 \leq i \leq d\right)$ is a velocity field, $\nu$ is the viscosity and $f(x, t)=-\nabla F(x, t)$ is a potential random forcing. Throughout the paper we assume that the random potential $F(x, t)$ is spatially periodic and smooth, and "white" in time:

$$
F(x, t)=\sum_{i=1}^{N} F_{i}(x) \dot{W}_{i}(t),
$$

where $F_{i}(x)$ are non-random $C^{\infty}$-smooth $\mathbb{Z}^{d}$-periodic potentials and $W_{i}(t)$ are independent white noises. The periodicity condition implies that effectively we consider the whole problem on the $d$-dimensional torus $\mathbb{T}^{d}=\mathbb{R}^{d} / \mathbb{Z}^{d}$. We shall only consider solutions which are provided by potential velocity fields: $u(x, t)=\nabla \phi(x, t)$. In this case a potential $\phi(x, t)$ satisfies the viscous Hamilton-Jacobi equation 


$$
\phi_{t}+\frac{1}{2}|\nabla \phi|^{2}+F(x, t)=\nu \Delta \phi .
$$

Although Burgers equation arises naturally in many different physical problems, (see $[5,16,4,13,3,12,1,2,11]$ ), we are primarily interested in the applications to hydrodynamics and the theory of turbulence. The main problems are then: existence and uniqueness of a stationary distribution for solutions to equation (1), and convergence of stationary distributions in the limit when viscosity tends to zero. It is important to mention, that the construction of the stationary distribution or the invariant measure corresponds to the limit $t \rightarrow \infty$. At the same time, the inviscid case, which is mostly interesting for turbulence theory, requires another limiting process $\nu \rightarrow 0$. It is tempting, first, to construct invariant measures $\mu_{\nu}$ for $\nu>0$, that is first to take limit as $t \rightarrow \infty$ and then to study a limit of $\mu_{\nu}$ as $\nu \rightarrow 0$. However, it is extremely difficult to control the last limiting process. Experience of the random Burgers equation suggests that it is more productive to consider from the very beginning $\nu=0$, construct unique invariant measure $\mu$, and only later prove that $\mu_{\nu} \rightarrow \mu$ as $\nu \rightarrow 0$.

Both problems which we formulated above were completely solved in [6] for the one dimensional case. However, the approach which was used in [6], besides being purely one-dimensional, is based on rather complicated probabilistic estimates. The main aim of the present paper is to show that the convergence of the invariant measures in the inviscid limit is a very general fact which follows from a simple conceptual argument.

The analysis in the inviscid case in [6], [14] and in this paper uses the connection between Burgers equation and Lagrangian dynamics. Very briefly we recall this connection. Let $L$ be the Lagrangian defined by

$$
L(x, v, t)=\frac{1}{2}|v|^{2}-F(x, t) .
$$

For a curve $\gamma:[a, b] \rightarrow M$ let

$$
A_{L}(\gamma)=\int_{a}^{b} L(\gamma(t), \dot{\gamma}(t), t) d t
$$

denote the action of the curve.

A curve $\gamma$ is called a minimizer if $A_{L}\left(\left.\gamma\right|_{[s, t]}\right) \leq A_{L}\left(\left.\beta\right|_{[s, t]}\right)$ for all subintervals $[s, t]$ and all curves $\beta$ such that $\beta(s)=\gamma(s)$ and $\beta(t)=$ $\gamma(t)$. A curve is called a backward one-sided minimizer if it is a minimizer in the interval $(-\infty, t)$, forward one-sided minimizer if it is a minimizer in the interval $(t, \infty)$, and global minimizer if it is minimizer in the whole line. It is proved in [6] that with probability 1 for all 
$t$ in $\mathbb{R}$ and for Lebesgue-almost all $x$ in $S^{1}$ there is a unique backward one-sided minimizer ending at $x$ at time $t$. It turns out that there exists a unique solution of the Burgers equation (1) which is extendible for all $t \in \mathbb{R}$, and this solution $u^{\omega}(x, t)$ is given by the velocity of $(x, t)$-backward one-sided minimizer at time $t$. Moreover, solutions $u^{\omega}(x, t)$ generate a unique stationary distribution $\mu$ for inviscid random forced Burgers equation. Notice, that $\omega$ above stands for a point of a probability space corresponding to the random force. It indicates a randomness of the solutions $u^{\omega}(x, t)$, i.e. their dependence on the concrete realization of the forcing term.

In the case of a positive viscosity, similar solutions $u_{\nu}^{\omega}(x, t)$ were previously constructed by Sinai in [18]. They also generate a unique stationary distribution $\mu_{\nu}$. In order to prove that $\mu_{\nu}$ converge to $\mu$, it was shown in [6] that $u_{\nu}^{\omega}(x, t)$ converges to $u^{\omega}(x, t)$ with probability 1 for almost all $x$ in $S^{1}$. As we have already mentioned above, the proof of this statement is quite complicated and relies on the HopfCole transformation $u_{\nu}=-2 \nu \nabla \log \phi$ which transforms equation (1) into a stochastic heat equation.

In the higher dimensional inviscid case, the "stationary" solutions $u^{\omega}(x, t)$ and a stationary distribution were constructed in [14] where the approach is based on a much more systematic use of the Lagrangian formalism and the Hamilton-Jacobi equation. More precisely, recall that the Hamiltonian $H$ associated to a convex Lagrangian $L$ is given by

$$
H(x, p)=\max _{v \in T_{x} M}[p \cdot v-L(x, v)] .
$$

The inviscid Hamilton-Jacobi has the following form

$$
\phi_{t}(x, t)+H(x, \nabla \phi, t)=0 .
$$

The connection with Lagrangian dynamics is given by the Lax formula solving the initial and final value problems. Solutions to the problems

$$
\begin{aligned}
& \phi_{t}+H(x, \nabla \phi, t)=0, t \in\left[T_{0}, T\right] \\
& \phi\left(\cdot, T_{0}\right)=g
\end{aligned}
$$

and

$$
\begin{aligned}
& \phi_{t}+H(x, \nabla \phi, t)=0, t \in\left[T_{0}, T\right] \\
& \phi(\cdot, T)=g
\end{aligned}
$$

are given respectively by the backwards Lax formula: 


$$
\phi(x, t)=\inf _{\gamma(t)=x}\left\{g(\gamma(0))+\int_{0}^{t} L(\gamma, \dot{\gamma}) d \tau\right\}
$$

and the forward Lax formula:

$$
\phi(x, t)=\sup _{\gamma(t)=x}\left\{g(\gamma(T))-\int_{t}^{T} L(\gamma, \dot{\gamma}) d \tau\right\} .
$$

In the special case when the Lagrangian $L(x, v, t)=\frac{1}{2}|v|^{2}-F(x, t)$ the inviscid Hamilton-Jacobi equation takes the simple form

$$
\phi_{t}(x, t)+\frac{1}{2}|\nabla \phi|^{2}+F(x, t)=0 .
$$

The solutions (7),(8) are unique viscosity solutions, and their spatial derivatives are the viscosity solutions to the inviscid Burgers equation with given initial or final values. Notice that the sign of the viscosity regularization term for the Hamilton-Jacobi equation strongly depends on whether we are dealing with the initial or final value problem. Namely, for the initial value problem (5) one has to consider a regularized problem

$$
\begin{aligned}
& \phi_{t}+H(x, \nabla \phi, t)=\nu \Delta \phi, t \in\left[T_{0}, T\right] \\
& \phi\left(\cdot, T_{0}\right)=g,
\end{aligned}
$$

where the viscosity $\nu$ is positive, while for the final value problem (5) the corresponding regularized problem

$$
\begin{aligned}
& \phi_{t}+H(x, \nabla \phi, t)=\nu \Delta \phi, t \in\left[T, T_{0}\right] \\
& \phi\left(\cdot, T_{0}\right)=g
\end{aligned}
$$

has negative viscosity $\nu=-\epsilon$. In both cases the viscosity solutions can be defined as a unique limit of the strong solutions to the systems (10), (11) as the viscosity $\nu$ tends to zero keeping the right sign.

Since we consider potential velocity fields and the forcing potentials are $\mathbb{Z}^{d}$-periodic, Burgers equation has a first integral which is the average velocity

$$
b=\int_{\mathbb{T}^{d}} u(x, t) d x
$$

Another manifestation of this first integral is provided by the following simple fact. For arbitrary $b \in \mathbb{R}^{d}$ define a set of potentials

$$
\Phi_{b}=\{\phi(x): \phi(x)=b \cdot x+\bar{\phi}(x)\}
$$


where $\bar{\phi}(x)$ are $\mathbb{Z}^{d}$-periodic. It is easy to see that the sets $\Phi_{b}$ are invariant under an evolution defined by the Hamilton-Jacobi equation (either viscous or inviscid). One can say that we have a one-parameter family of problems which are parametrized by a parameter $b$. We shall see below that due to the randomness of the forcing term, all results and their proofs do not depend on the value of $b$. Therefore, for simplicity, we give proofs in the case $b=0$, which corresponds to $\mathbb{Z}^{d}$-periodic solutions to the Hamilton-Jacobi equation.

It was proved in [14] that with probability 1 , for all $b \in \mathbb{R}^{d}$, there exist unique backward and forward viscosity solutions to the inviscid Hamilton-Jacobi equation which are extendible for all $t \in \mathbb{R}$. We shall denote those solution $\psi_{-}^{\omega, b}(x, t)$ and $\psi_{+}^{\omega, b}(x, t)$ respectively. It was also shown that, independently of the initial and final conditions, provided that they belong to $\Phi_{b}$, the solutions $\phi(x, t)$ of problems $(5),(6)$ tend to $\psi_{-}^{\omega, b}(x, t), \psi_{+}^{\omega, b}(x, t)$ respectively in a limit when $T_{0} \rightarrow-\infty$ or $T \rightarrow \infty$. The same convergence holds for spatial derivatives which give corresponding solutions to the inviscid Burgers equation.

Since backward and forward solutions are obviously related by the transformation $t \rightarrow-t$, it is enough to study only one of the two cases. In this paper the proofs are presented for the case of forward solutions corresponding to the final value problem (11) regularized by the negative viscosity term. Solutions to this problem are given by the stochastic Lax formula (see [10])

$$
\phi(x, t)=\sup _{u} E\left[g\left(x^{u}\left(T_{0}\right)\right)-\int_{t}^{T_{0}} L^{\omega}\left(x^{u}(\tau), u(\tau)\right) d \tau\right],
$$

where $x^{u}$ is a solution of the stochastic differential equation

$$
d x^{u}=u d t+\sqrt{2 \epsilon} d B, x^{u}(t)=x,
$$

$u$ is a bounded progressively measurable control and $\epsilon=-\nu$. Notice that $d B=\left(d B^{1}, \ldots, d B^{d}\right)$ in $(15)$ is an "external" white noise vector which has no connection with the "internal" white noises $\dot{W}_{i}$ in the expression (2) for the random potential $F(x, t)$. The "external" white noise is used only to write the formula (14) for a solution $\phi(x, t)$ where the expectation is taken with respect to $d B$ and no average is done with respect to $W$. We remark that due to a quite irregular character of the time dependence of $F(x, t)$, the stochastic integral in (14) has to be correctly defined. We shall do it in the next section.

We are now ready to formulate the main results of the paper. Fix the value of the first integral $b \in \mathbb{R}^{d}$ and assume that the initial and final values for the problems (10), (11) belong to $\Phi_{b}$. Then, exactly as in the 
inviscid situation (see [14]), in the case of positive viscosity solutions to the initial value problem (10) converge in a limit $T_{0} \rightarrow-\infty$ to a unique (up to an additional constant) solution to the viscous Hamilton-Jacobi equation

$$
\phi_{t}+H(x, \nabla \phi, t)=\nu \Delta \phi
$$

which is extendible for all times. Similarly, in the case of negative viscosity solutions to the final value problem (11) converge in a limit $T \rightarrow \infty$. More precisely, the following theorem holds.

\section{Theorem 1.}

1. With probability 1 , for all $\nu \neq 0$ and all $b \in \mathbb{R}^{d}$, there exists $a$ unique (up to an additional constant) solution $\psi_{\nu}^{\omega, b}$ of (16) which is extendible for all times.

2. With probability 1 for a fixed $\nu \neq 0, b \in \mathbb{R}^{d}$ solutions $\phi(x, t)$ to the initial value problem (10) (in the case of positive viscosity) or to the final value problem (11) (in the case of negative viscosity) converge (up to an additional constant) to $\psi_{\nu}^{\omega, b}$ in the uniform topology as $T_{0} \rightarrow-\infty$ or $T \rightarrow \infty$. Moreover, $\nabla \phi(x, t)$ converge to $\nabla \psi_{\nu}^{\omega}$ in the same limit.

Notice that the potentials $\psi_{\nu}^{\omega, b}$ are defined up to an additional constant. Let us fix an arbitrary point $x_{0} \in \mathbb{T}^{d}$ and assume that $\psi_{\nu}^{\omega, b}\left(x_{0}\right)=$ 0 . Since the stochastic Lax formula (14) roughly speaking tends to the Lax formula (8) as $\nu$ tends to zero, we get $\psi_{\nu}^{\omega, b} \rightarrow \psi_{-,+}^{\omega, b}$ in a viscosity limit depending on the sign of the viscosity. Indeed, it is easy to show that functions given by the Lax formulae are Lipschitz. Moreover from control theory estimates, as will be discussed in the next section, this Lipschitz constant is uniform in $\nu$. Then according to Arzela-Ascoli theorem there exist a uniformly convergent subsequence $\psi_{\nu_{j}}^{\omega}$. It is easy to show that the limiting function is a viscosity solution to the inviscid Hamilton-Jacobi equation: backward when $\nu>0$ or forward if $\nu<0$. This together with the uniqueness of viscosity solutions implies the following result.

Theorem 2. With probability 1, as $\nu \rightarrow 0$ without changing sign, the solutions $\psi_{\nu}^{\omega, b}$ of (16) converge uniformly to the unique (up to an additional constant) backward (in the case $\nu>0$ ) or forward (in the case $\nu<0)$ viscosity solution $\psi_{-,+}^{\omega, b}$ of the inviscid Hamilton-Jacobi equation (4).

The solutions $\psi_{\nu}^{\omega, b}$ of the Hamilton-Jacobi equation are spatially differentiable. The gradient of these solutions satisfy the viscous Burgers equation. However, the convergence of $\psi_{\nu}^{\omega, b}$ to $\psi_{-,+}^{\omega, b}$ cannot be 
uniform in $C^{1}$-topology. In fact, the inviscid solutions $\psi_{-,+}^{\omega, b}$ are only Lipschitz continuous and the corresponding solutions to the inviscid Burgers equation $u_{-,+}^{\omega, b}=\nabla \psi_{-,+}^{\omega, b}$ are only defined at the points of differentiability of $\psi_{-,+}^{\omega, b}(x, t)$. Such points of differentiability are exactly the uniqueness points for the backward and forward one-sided minimizer starting at time $t$ at this point (see Fathi [9]). Notice, that the Lagrangian for one-sided minimizers depends on $b$ ([14]).

Recall that a function $f: \mathbb{R}^{n} \rightarrow \mathbb{R}$ is called semiconvex if there exists a positive constant $C$ such that

$$
f(x+h)-2 f(x)+f(x-h) \geq-C h^{2} .
$$

Equivalent one can say that $f-C x^{2}$ is a concave function.

It is easy to see that any solution given by the Lax formula is semiconcave, and, in fact, the semiconcavity constant can be chosen uniformly in $\nu$ (Lemma 6). On the other hand, it is almost obvious that if $\xi_{n} \rightarrow \xi_{0}$ is a convergent sequence of planes of support of a convergent sequence $f_{n} \rightarrow f_{0}$ of concave functions, then $\xi_{0}$ is a plane of support of $f_{0}$ (see Lemma 10 below). These two simple facts imply the following result:

Theorem 3. With probability 1, in the limit of vanishing $\nu$, at all points of differentiability of $\psi_{-,+}^{\omega, b}(x, t)$ the solution $u_{\nu}^{\omega, b}=\nabla \psi_{\nu}^{\omega, b}$ converges pointwise to $u_{-}^{\omega, b}=\nabla \psi_{-}^{\omega, b}$ (in the case of positive viscosity) and to $u_{+}^{\omega, b}=\nabla \psi_{+}^{\omega, b}$ (in the case of negative viscosity).

Finally, we formulate the results on the convergence of stationary distributions. Denote by $(\Omega, \mathcal{B}, P)$ the probability space corresponding to the stationary process $\dot{W}_{i}(t), 1 \leq i \leq N$. The measurable mappings

$$
\begin{aligned}
& \omega \mapsto \psi_{\nu}^{\omega, b}(x, 0)-b \cdot x, \omega \mapsto \nabla \psi_{\nu}^{\omega, b}(x, 0), \\
& \omega \mapsto \psi_{-}^{\omega, b}(x, 0)-b \cdot x, \omega \mapsto \nabla \psi_{-}^{\omega, b}(x, 0)
\end{aligned}
$$

generate four probability measures in functional spaces which we denote by $m_{\nu}^{b}, \mu_{\nu}^{b}, m^{b}, \mu^{b}$ respectively. The measure $m^{b}$ is defined in the space $C\left(\mathbb{T}^{d}\right) / \mathbb{R}$ of equivalence classes of continuous functions on the torus $\mathbb{T}^{d}$ up to an additional constant. This space is equipped with the uniform topology which defines the corresponding Borel $\sigma$-algebra. The measure $\mu^{b}$ is naturally defined on a subset $\mathcal{U}_{b}$ of the measure space corresponding to $L^{p}\left(\mathbb{T}^{d}, d x\right), p \geq 1$ (see [14]). The set $\mathcal{U}_{b}$ consists of all functions of the form $b+\nabla \phi(x)$ for some Lipschitz function $\phi(x)$ on $\mathbb{T}^{d}$. It was proven in [14] that for fixed $b \in \mathbb{R}^{d}$ the measures $m^{b}$ and $\mu^{b}$ are unique stationary distribution for the Markov processes corresponding to random Hamilton-Jacobi and Burgers equations. Although the 
measures $m_{\nu}^{b}$ and $\mu_{\nu}^{b}$ are supported in the set of smooth functions, it is convenient to consider them on the bigger spaces $C\left(\mathbb{T}^{d}\right) / \mathbb{R}$ and $\mathcal{U}_{b}$.

Theorem 4. Assume that $\nu$ is positive.

- For fixed $b \in \mathbb{R}^{d}$ the measures $m_{\nu}^{b}$ and $\mu_{\nu}^{b}$ are the unique stationary distributions for the Markov processes corresponding to the viscous random Hamilton-Jacobi and Burgers equations.

- In the limit when $\nu$ tends to zero the measures $m_{\nu}^{b}$ and $\mu_{\nu}^{b}$ converge weakly to $m^{b}$ and $\mu^{b}$ respectively.

Obviously, similar statements hold in the case of negative $\nu$. However, in this case one has to define the corresponding Markov processes in inverse time.

In Section 2 we define the stochastic integral in (14) and prove Theorem 1. Theorems 2, 3 and 4 are proven in Section 3. We finish with concluding remarks in the last Section.

\section{Existence And uniqueness of SOlutions in the CASE OF POSITIVE VISCOSITY}

In this section we follow very closely the arguments in [14] which are originally inspired by [6]. The viscosity $\nu<0$ and the average velocity $b=0$ are fixed and will be omitted from all the notation. As above, denote $\epsilon=-\nu$. For simplicity we set $b=0$. It will be clear that the proofs do not change for other values of $b$.

Firstly, we will define the action:

$$
E\left[g\left(x^{u}\left(T_{0}\right)\right)-\int_{t}^{T_{0}} L\left(x^{u}(\tau), u(\tau)\right) d \tau\right]
$$

for the stochastic Lax formula (14) to make sense. Since one takes expectation in (19) it is easy to see that it is possible to use integration by parts. Applying the Ito formula

$$
d F\left(x^{u}(t)\right)=\nabla F\left(x^{u}(t)\right) \cdot u(t) d t+\epsilon \Delta F\left(x^{u}(t)\right) d t+\nabla F\left(x^{u}(t)\right) \cdot d W(t)
$$


we have

$$
\begin{array}{rl}
\phi(x, t) & =E\left[g\left(x^{u}\left(T_{0}\right)\right)-\int_{t}^{T_{0}} L\left(x^{u}(\tau), u(\tau)\right) d \tau\right] \\
=E & E\left(x^{u}\left(T_{0}\right)\right) \\
-E & {\left[\int _ { t } ^ { T _ { 0 } } \left[\frac{1}{2}|u(\tau)|^{2}\right.\right.} \\
& \left.\left.+\sum_{i=1}^{N}\left(\nabla F_{i}\left(x^{u}(\tau)\right) \cdot u(\tau)+\epsilon \Delta F_{i}\left(x^{u}(\tau)\right)\right)\left(W_{i}(\tau)-W_{i}(t)\right)\right] d \tau\right] \\
- & \sum_{i=1}^{N} F_{i}(x)\left(W_{i}(t)-W_{i}\left(T_{0}\right)\right),
\end{array}
$$

where we have used the following relation

$$
E \sum_{i=1}^{N} F_{i}\left(x^{u}(t)\right)\left(W_{i}(t)-W_{i}\left(T_{0}\right)\right)=\sum_{i=1}^{N} F_{i}(x)\left(W_{i}(t)-W_{i}\left(T_{0}\right)\right) .
$$

Notice that the contribution of the last term in (20) vanishes since its expected value is zero. There is also another method to derive formula (21) which was suggested to us by P. Souganidis. This method does not use Ito calculus. Starting with the random forced HamiltonJacobi equation (3) with $F(x, t)$ given by (2) one gets after a simple transformation

$$
\varphi(x, t)=\phi(x, t)+\sum_{i=1}^{N} F_{i}(x)\left(W_{i}(t)-W_{i}\left(T_{0}\right)\right)
$$

the following equation:

$$
\begin{aligned}
\varphi_{t}+ & \frac{1}{2}\left|\nabla \varphi-\sum_{i=1}^{N} \nabla F_{i}(x)\left(W_{i}(t)-W_{i}\left(T_{0}\right)\right)\right|^{2} \\
& -\epsilon \sum_{i=1}^{N} \Delta F_{i}(x)\left(W_{i}(t)-W_{i}\left(T_{0}\right)\right)=\nu \Delta \varphi
\end{aligned}
$$

which corresponds to a Hamiltonian 


$$
\begin{gathered}
H(p, x, t)=\frac{1}{2}\left|p-\sum_{i=1}^{N} \nabla F_{i}(x)\left(W_{i}(t)-W_{i}\left(T_{0}\right)\right)\right|^{2} \\
-\epsilon \sum_{i=1}^{N} \Delta F_{i}(x)\left(W_{i}(t)-W_{i}\left(T_{0}\right)\right)
\end{gathered}
$$

and a Lagrangian

$$
\begin{aligned}
& L(v, x, t)=\frac{1}{2}|v|^{2}+v \cdot \sum_{i=1}^{N} \nabla F_{i}(x)\left(W_{i}(t)-W_{i}\left(T_{0}\right)\right) \\
& +\epsilon \sum_{i=1}^{N} \Delta F_{i}(x)\left(W_{i}(t)-W_{i}\left(T_{0}\right)\right) .
\end{aligned}
$$

It is easy to see that the last formula immediatly implies relation (21) for $\phi(x, t)$.

We shall consider the stochastic Lax formula as a non linear operator acting on the space of continuous functions $C\left(\mathbb{T}^{d}\right)$. Define, for every $s_{1}<s_{2}$ and any $\omega$ corresponding to a continuous realization of the white noises $W_{i}, 1 \leq i \leq N$, the transformation

$$
K_{s_{1}, s_{2}}^{\omega} \phi(x)=\sup _{u} E\left[g\left(x^{u}\left(s_{2}\right)-\int_{s_{1}}^{s_{2}} L^{\omega}\left(x^{u}(\tau), u(\tau)\right) d \tau\right],\right.
$$

where supremum is taken over all bounded progressively measurable controls $u, E[\cdot]$ is defined by $(21)$ and $x^{u}\left(s_{1}\right)=x$. In order to prove the existence and uniqueness of the solutions to the Hamilton-Jacobi equation we will show that there is a unique (up to an additive constant) $\phi^{\omega}(x, t), t \in \mathbb{R}$, such that for all $s_{1}<s_{2}$

$$
K_{s_{1}, s_{2}}^{\omega} \phi\left(\cdot, s_{2}\right)=\phi\left(\cdot, s_{1}\right)
$$

We begin with the following lemmas.

Lemma 1. With probability 1 the operator $K_{s, t}^{\omega}$ has the following properties

(1) The semigroup property: for all $s_{1}<s_{2}<s_{3}$ we have

$$
K_{s_{1}, s_{3}}^{\omega}=K_{s_{1}, s_{2}}^{\omega} \circ K_{s_{2}, s_{3}}^{\omega} .
$$

(2) The weak contraction property

$$
\left\|K_{s_{1}, s_{2}}^{\omega} \phi_{1}-K_{s_{1}, s_{2}}^{\omega} \phi_{2}\right\| \leq\left\|\phi_{1}-\phi_{2}\right\| .
$$


(3) For any constant $c$,

$$
K_{s_{1}, s_{2}}^{\omega}(\phi+c)=K_{s_{1}, s_{2}}^{\omega}(\phi)+c .
$$

\section{Proof:}

The first statement follows from the fact that $K_{s_{1}, s_{2}}^{\omega} \phi$ gives the solution to the Cauchy problem (11).

To prove the second statement consider two continuous functions $\phi_{1}, \phi_{2}$. Let $u$ be a $\delta$-optimal control for $\phi_{2}$, i.e., a control such that such that

$$
K_{s_{1}, s_{2}}^{\omega} \phi_{2}(x) \geq E\left[\phi_{2}\left(x^{u}\left(s_{2}\right)\right)-\int_{s_{1}}^{s_{2}} L^{\omega}\left(x^{u}(\tau), u(\tau)\right) d \tau\right]-\delta .
$$

By definition

$$
K_{s_{1}, s_{2}}^{\omega} \phi_{1}(x) \leq E\left[\psi_{1}\left(x^{u}\left(s_{2}\right)\right)-\int_{s_{1}}^{s_{2}} L^{\omega}\left(x^{u}(\tau), u(\tau)\right) d \tau\right] .
$$

Subtracting we get

$$
K_{s_{1}, s_{2}}^{\omega} \phi_{1}(x)-K_{s_{1}, s_{2}}^{\omega} \phi_{2}(x) \leq E\left[\phi_{1}\left(x^{u}(t)\right)-\phi_{2}\left(x^{u}(t)\right)\right]+\delta .
$$

Since $\delta$ is arbitrary small and $E\left(\phi_{1}\left(x^{u}(t)\right)-\phi_{2}\left(x^{u}(t)\right)\right) \leq\left\|\phi_{1}-\phi_{2}\right\|_{C}$, we get

$$
K_{s_{1}, s_{2}}^{\omega} \phi_{1}(x)-K_{s_{1}, s_{2}}^{\omega} \phi_{2}(x) \leq\left\|\phi_{1}-\phi_{2}\right\|_{C} .
$$

Interchanging $\phi_{1}$ and $\phi_{2}$ we obtain

$$
\left|K_{s_{1}, s_{2}}^{\omega} \phi_{1}(x)-K_{s_{1}, s_{2}}^{\omega} \phi_{2}(x)\right| \leq\left\|\phi_{1}-\phi_{2}\right\|_{C} .
$$

The last statement of the lemma is obvious.

Consider the Cauchy problem (11) with final condition $g$ at time $n$. Denote by $c_{i}(n, g)$ the Lipschitz constant in the spacial variable of the solution at time $i$ and by $c(g)$ the Lipschitz constant of $g$.

Lemma 2. Assume that $\epsilon \leq 1$, then

(1) For almost all $\omega$ there exist a random constant $c(\omega)$ such that $c_{0}(n, \psi) \leq c(\omega)$ for every $\psi$, if $n$ is large enough.

(2) There exists a constant $C$ and a set $\Sigma$ of positive measure such that for all $\omega \in \Sigma, c_{0}(n) \leq C$ for all $n$ large enough.

Remark 1. How large is $n$ depends essentially on the Lipschitz constant of $\psi$. In the non viscous case, we can use $n=1$.

Before prooving the lemma we first need an estimate of $c_{0}(1, g)$ in terms of $c(g)$.

Lemma 3. There exist a $U(\omega)$ such that

$$
c_{0}(1, g) \leq U+U \sqrt{c(g)} \text {. }
$$


Proof: Recall the action

$$
\begin{aligned}
& E\left[g\left(x^{u}\left(T_{0}\right)\right)-\int_{t}^{T_{0}} L\left(x^{u}(\tau), u(\tau)\right) d \tau\right] \\
& =E g\left(x^{u}\left(T_{0}\right)\right)-E \int_{t}^{T_{0}}\left[\frac{1}{2}|u(\tau)|^{2}\right. \\
& \left.\quad+\sum_{i=1}^{N}\left(\nabla F_{i}\left(x^{u}(\tau)\right) \cdot u(\tau)+\epsilon \Delta F_{i}\left(x^{u}(\tau)\right)\right)\left(W_{i}(\tau)-W_{i}(t)\right)\right] d \tau \\
& -\sum_{i=1}^{N} F_{i}(x)\left(W_{i}(t)-W_{i}\left(T_{0}\right)\right) .
\end{aligned}
$$

Denote

$M=\max _{x \in \mathbb{T}^{d}} \max _{1 \leq i \leq N}\left\{\left|\nabla F_{i}(x)\right|,\left|\Delta F_{i}(x)\right|,\left\|D^{2} \Delta F_{i}(x)\right\|,\left\|D^{2} F_{i}(x)\right\|,\left\|D^{3} F_{i}(x)\right\|\right\}$,

and

$$
V(\omega)=\max _{\tau, s \in[0,1]} \max _{1 \leq i \leq N}\left|W_{i}(s)-W_{i}(\tau)\right| .
$$

Let $x$ and $y$ be two points and $u$ an optimal control for $x$ and $g$. Define $\bar{u}=u+x-y$, then we have

$$
\begin{aligned}
& \phi(x, 0)-\phi(y, 0) \leq \\
& \quad \leq E\left(g\left(x^{u}(1)\right)\right)-E\left(g\left(y^{\bar{u}}(1)\right)\right) \\
& \quad+E \int_{0}^{1} \frac{1}{2}|\bar{u}(\tau)|^{2}-E \int_{0}^{1} \frac{1}{2}|u(\tau)|^{2} \\
& \quad+\left(\sum_{i=1}^{N} \nabla F_{i}\left(y^{\bar{u}}(\tau)\right) \cdot \bar{u}(\tau)-\nabla F_{i}\left(x^{u}(\tau)\right) \cdot u(\tau)\right)\left(W_{i}(\tau)-W_{i}(t)\right) \\
& \quad+\epsilon\left(\sum_{i} \Delta F_{i}\left(y^{\bar{u}}(\tau)\right)-\Delta F_{i}\left(x^{\bar{u}}(\tau)\right)\right)\left(W_{i}(\tau)-W_{i}(t)\right) \\
& \quad-\epsilon \sum_{i=1}^{N}\left(F_{i}(x)-F_{i}(y)\right)\left(W_{i}(0)-W_{i}(1)\right)
\end{aligned}
$$

Then $(i)$ vanishes by the choice of the control. Also (ii) is bounded by

$$
|x-y|^{2}+\int_{0}^{1}|u||x-y|
$$


the third term, (iii), by

$$
N M V|x-y|+N M V \int_{0}^{1}|u||x-y|,
$$

and $(i v)$ and $(v)$ by

$$
\epsilon 3 N M V|x-y|
$$

Denote by $\ell=\int_{0}^{1}|u|$, then

$$
c_{0}(1, g) \leq 2 \operatorname{diam}\left(\mathbb{T}^{d}\right)+\ell+\epsilon 2 N M V+N M V \ell,
$$

or, with a large enough constant $k$ that only depends on the dimension, the number of potentials and the $C^{4}$ norm of the potentials,

$$
c_{0}(1, g) \leq k+k V+\ell+k V \ell .
$$

We can estimate $\ell$ in terms of the Lipschitz constant of the final value, $c(g)$.

Without loss of generality we can assume that $g\left(x_{0}\right)=0$. Then using no control whatsoever

We get that $\phi(x, 0) \geq-c(g)-N M V$. On the other hand using the optimal control we get

$$
\phi(x, 0) \leq c(g)-\int_{0}^{1}|u|^{2}+N M V \int_{0}^{1}|u|+\epsilon 2 N M V .
$$

Using Schwarz inequality we obtain

$$
\ell^{2}-N M V l \leq 2 c(g)+3 N M V
$$

So

$$
q \ell \leq \frac{1}{2}\left(N M V+\sqrt{N^{2} M^{2} V^{2}+4(2 c(g)+3 N M V)}\right) .
$$

Thus, for a large enough constant $s$ that only depends on the dimension, the number of potentials and the $C^{4}$ norm of the potentials we have

$$
\ell \leq s+s V+s \sqrt{c(g)}
$$

The previous inequality combined with (31) yields

$$
c_{0}(1, g) \leq k+s+(k+s+k s) V+k s V^{2}+(s+k V) \sqrt{c(g)} .
$$

Let

$$
U=\max \left\{k+s+(k+s+k s) V+k s V^{2},(s+k V)\right\} .
$$

Then the above inequality reads

$$
c_{0}(1, g) \leq U+U \sqrt{c(g)}
$$


Let $\Omega$ be the probability space of the Brownian motion $W_{i}$. The functions $V$ and $U$ defined as before. Let $\sigma$ denote the time one shift. Let $V_{n}=V \circ \sigma^{n}$ and $U_{n}=U \circ \sigma^{n}$.

Lemma 4. If $U_{j}<2^{j}$ then the set $\left\{c_{0}(n, g): n \in N\right\}$ is bounded.

Proof: The previous calculations show that the numbers $c_{i}(n, g)$ satisfy the following recursive inequalities

$$
c_{j-1}(n, g) \leq U_{j} \sqrt{c_{j}(n, g)}+U_{j}
$$

In the rest of the lemma we drop from the notation of the Lipschitz constants the dependence on $g$ and the final time $n$. So we have

$$
c_{n-1} \leq U_{n} \sqrt{c_{n}}+U_{n}
$$

Then

$$
\begin{gathered}
c_{n-1} \leq(1+\sqrt{c}) U_{n}, \\
c_{n-2} \leq U_{n-1}\left(U_{n}\right)^{\frac{1}{2}}+U_{n-1} \\
c_{n-3} \leq U_{n-2}\left(U_{n-1}\left((1+\sqrt{c}) U_{n}\right)^{\frac{1}{2}}+U_{n-1}\right)^{\frac{1}{2}}+U_{n-2} \leq \\
\leq U_{n-2}\left(2 U_{n-1}\left((1+\sqrt{c}) U_{n}\right)^{\frac{1}{2}}\right)^{\frac{1}{2}}+U_{n-2}= \\
\left.2^{\frac{1}{2}} \cdot(1+\sqrt{c})^{\frac{1}{2^{2}}} U_{n-2}\left(U_{n-1}\right)^{\frac{1}{2}}\left(U_{n}\right)^{\frac{1}{2^{2}}}\right)+U_{n-2}
\end{gathered}
$$

By induction we get

$$
c_{0}(n, g) \leq U_{1} U_{2}^{\frac{1}{2}} U_{3}^{\frac{1}{2^{2}}} \ldots U_{n}^{\frac{1}{2^{n-1}}} 2 \cdot 2^{\frac{1}{2}} \cdot 2^{\frac{1}{2^{2}}} \ldots(1+\sqrt{c})^{\frac{1}{2^{n}}}+U_{1} .
$$

We have then that

$$
\begin{gathered}
\log \frac{\left(c_{0}(n, g)-U_{1}\right)}{(1+\sqrt{c})^{\frac{1}{2^{n}}}}=\sum \log \left(2 U_{i}\right)^{\frac{1}{2^{i-1}}} \leq \\
\leq \sum \frac{\log \left(2^{i+1}\right)}{2^{i-1}}= \\
=\sum \frac{i+1 \log (2)}{2^{i-1}},
\end{gathered}
$$

which is convergent in $n$. Since $(1+\sqrt{c})^{\frac{1}{2^{n}}}$, converges to 1 we see that the bound for $c_{0}(n, g)$ is independent of $c(g)$.

Corollary 1. If $U_{j}<2^{j}$ for all but a finite number of indices then there is a random constant $C(\omega)$ such that $c_{0}(n) \leq C(\omega)$. 
Proof: Let $T$ be such that $U_{j} \leq 2^{j}$ for every $j$ bigger than $T$. Then for $\tilde{\omega}=\sigma_{M} \omega$ we have that $U_{j}(\tilde{\omega}) \leq 2^{M} \cdot 2^{j}$

The same calculation of the previous lemma gives rise to a factor of $\prod_{i=1}^{n} 2^{\frac{M}{2^{i}}}$. So, the Lipschitz constant at time $T$ will be bounded, therefore also is the Lipschitz constant a time zero also. The bound will depend on $T$ and on the behavior until time $T$.

Lemma 5. The set of $\omega$ in $\Omega$ such that there are only a finite number of $j$ such that $U_{j}>2^{j}$ has full measure.

Proof: The proof of this lemma is a consequence of the integrability of $U$. Let $F_{j}=U^{-1}\left(2^{j}, \infty\right)$ and $A_{j}=\cup_{i=0}^{j} \sigma^{-i} F_{j}$. Define

$M=\left\{\omega\right.$ : there exist an infinite number $n$ such that $\left.U_{n}>2^{n}\right\}$.

Then

$$
M=\cap_{j=1}^{\infty} \cup_{l=j}^{\infty} A_{l} .
$$

Let $L=\int U$ then the measure of $F_{j}$ is less or equal than $\frac{L}{2^{j}}$. Therefore the measure of $A_{j}$ is less or equal than $\frac{L j}{2^{j}}$. It follows that

$$
\mu\left(\cup_{l=j}^{\infty} A_{l}\right) \leq \sum_{j=l}^{\infty} \frac{L j}{2^{j}} .
$$

The sum is convergent so the tails converge to zero, hence the measure of $M$ is equal to zero.

The proof of lemma 2 is now an immediate consequence of the previous lemma and the corollary. For the proof of theorem 3 we will need a uniform estimate for the semiconcavity constant of the solution:

Lemma 6. Given a positive constant $C$, there exists, for almost all $\omega$, a random constant $S(\omega)>0$, such that the semiconcavity constant of $\phi$, the solution of problem 11 is less than $S(\omega)$, provided $c(g) \leq C$.

Proof: Consider an increment $h$ and define curves $x_{ \pm}(\tau)=x^{u}(\tau) \pm$ $y(\tau)$, where $y(\tau)=-h \tau$. The curves $x_{ \pm}(\tau)$ correspond to the admissible controls $u(\tau) \mp h$ which gives

$$
\phi(x \pm h, 0) \geq E\left(g\left(x^{u}(1)\right)-E \int_{0}^{1} L\left(x_{ \pm}(\tau), u(\tau) \mp h\right) d \tau .\right.
$$

Since

$$
\phi(x, 0)=E\left(g\left(x^{u}(1)\right)-E \int_{0}^{1} L\left(x^{u}(\tau), u(\tau)\right) d \tau\right.
$$


and recalling the estimate (32):

$$
\int_{0}^{1}|u(\tau)| \leq \frac{1}{2}\left[N M V+\sqrt{N^{2} M^{2} V^{2}+4(2 c(\phi(\cdot), 1)+3 N M V)}\right]=A
$$

we get from (21):

$$
\begin{aligned}
& \phi(x+h,-1)-2 \phi(x,-1)+\phi(x+h,-1) \\
& \geq-|h|^{2}\left(1+2 M V(\omega) N+\frac{1}{2} M V(\omega) A N\right) .
\end{aligned}
$$

Since Lipschitz constants at time one are bounded, we obtain semiconcavity.

In what follows we estimate the behavior of solutions for large time when the potentials are small. The content of the following lemma may be roughly summarized by saying that in a sufficiently long time interval in which the potentials are small, the solution $\phi$ is transformed to a function close to a constant. Before giving a precise statement we need the following definition. We denote by

$$
V\left(\omega, s_{1}, s_{2}\right)=\max _{\tau, s \in\left[s_{1}, s_{2}\right]} \max _{1 \leq i \leq N}\left|W_{i}(s)-W_{i}(\tau)\right|
$$

For simplicity we will not write explicitly the dependence of $V$ with respect to its arguments.

Lemma 7. Let $\delta>0$ and $C>0$ be given. Then there exist $T>0$ and $\alpha>0$ such that, if

(1) $V\left(s_{1}, s_{2}\right) \leq \alpha$

(2) $s_{2}-s_{1}=T$, and

(3) $c(\phi) \leq C$,

then

$$
c_{s_{1}}\left(s_{2}, \phi\right) \leq \delta
$$


Proof We will use the Lax formula and perform similar computations to the ones in lemma 3

$$
\begin{aligned}
\left|\varphi\left(y, s_{1}\right)-\varphi\left(x, s_{1}\right)\right| & \\
\leq & \left|E\left(\phi\left(y^{\bar{u}}, s_{2}\right)\right)-E\left(\phi\left(x^{u}, s_{2}\right)\right)\right| \\
+ & \left.\left|E \int_{s_{1}}^{s_{2}} \frac{1}{2} \overline{\mid}\right| u\right|^{2}-\frac{1}{2}|u|^{2} \mid \\
+ & \mid E \int_{s_{1}}^{s_{2}} \sum_{i=1}^{N}\left(\nabla F_{i}\left(x^{\bar{u}}(\tau)\right) \cdot \bar{u}\right. \\
& \left.\quad-\nabla F_{i}\left(x^{u}(\tau)\right) \cdot u\right)\left(W_{i}(\tau)-W_{i}(t)\right) d \tau \mid \\
+ & \mid E \int_{s_{1}}^{s_{2}} \sum_{i=1}^{N} \epsilon\left[\Delta F_{i}\left(x^{\bar{u}}(\tau)\right)\right. \\
& \left.\quad-\Delta F_{i}\left(y^{u}(\tau)\right)\right]\left(W_{i}(\tau)-W_{i}(t)\right) d \tau \mid \\
+ & \left|\sum_{i=1}^{N}\left(F_{i}(x)-F_{i}(y)\right)\left(W_{i}\left(s_{1}\right)-W_{i}\left(s_{2}\right)\right)\right|
\end{aligned}
$$

were $u$ is the optimal control with initial condition $x$ and the modified control $\bar{u}=u+\frac{x-y}{T}$, for $y$.

The first term vanishes if we choose, as in lemma 3 ,

$$
\bar{u}=u+\frac{x-y}{T} .
$$

As for the other terms, $(i v)$ and $(v)$ can easily be estimated:

$$
(i v) \leq M N V T|x-y|,
$$

and

$$
(v) \leq M N V \cdot|x-y|
$$

Before estimating $(i i)$ and $(i i i)$, we need the following estimate.

Lemma 8. If $V$ is sufficiently small so that

$$
M N V \leq \frac{1}{T^{1 / 2}},
$$

then, there is a positive number $\lambda$ not depending on $T$ such that

$$
\ell=\int_{s_{1}}^{s_{2}}|u| \leq \lambda \sqrt{T}
$$


Proof: By Schwarz inequality we have

$$
\begin{aligned}
\ell & \leq\left(\int_{s_{1}}^{s_{2}}|u|^{2} d t\right)^{1 / 2}\left(\int_{s_{1}}^{s_{2}} d t\right)^{1 / 2} \\
& \leq T^{1 / 2}\left(\int_{s_{1}}^{s_{2}}|u|^{2} d t\right)^{1 / 2}
\end{aligned}
$$

Using Lax formula again

$$
\phi\left(x, s_{1}\right) \leq c(\phi)-\int_{s_{1}}^{s_{2}}|u|^{2}+N M V \ell+2 \epsilon N M V,
$$

and putting these two estimates together

$$
\phi\left(x, s_{1}\right) \leq c(\phi)-\frac{\ell^{2}}{T}+N M V \ell+2 \epsilon N M V .
$$

Therefore we have

$$
\frac{\ell^{2}}{T}-N M V \ell \leq 2 \epsilon N M V+c(\phi)+\operatorname{diam}\left(\mathbb{T}^{d}\right) c_{s_{1}}\left(s_{2}, \phi\right) .
$$

From the previous inequality we obtain a bound for $\ell$ as

$$
\ell \leq \frac{1}{2}\left(1+\sqrt{1+4\left(2 \epsilon M N V+c(\phi)+\operatorname{diam}\left(\mathbb{T}^{d}\right) c_{s_{1}}\left(s_{2}, \phi\right)\right)}\right) \sqrt{T}
$$

From this bound we see that for $T$ large such that

$$
N M V T \leq \lambda T^{1 / 2}
$$

and using this estimate in $(i)$ and $(i i)$,

$$
\begin{aligned}
(i) & \leq \frac{|x-y|^{2}}{T^{2}}+\int_{0}^{T} u \cdot \frac{(x-y)}{T} d \tau \\
& \leq \frac{c_{1}}{T^{2}}+c_{2} \frac{\lambda}{T^{1 / 2}}
\end{aligned}
$$

For the term

$$
\begin{aligned}
(i i) \leq & M N V|x-y|+\int_{s_{1}}^{s_{2}}|u| \cdot M N V \frac{|x-y|}{T} \\
& \leq M N V|x-y|+M N V \frac{|x-y|}{\sqrt{T}} .
\end{aligned}
$$

Putting together the estimates for $(i)$ to $(v)$ we get $\phi\left(x, s_{1}\right)-\phi\left(y, s_{2}\right) \leq\left(\frac{\lambda}{\sqrt{T}}+\frac{|x|+|y|}{T}+M N V+\frac{M N V}{\sqrt{T}}+M N V T\right)|x-y|$. 
So the Lipschitz constant $c_{s_{1}}\left(s_{2}, \phi\right)$ satisfies

$$
c_{s_{1}}\left(s_{2}, \phi\right) \leq \frac{\lambda}{\sqrt{T}}+\frac{x+y}{T}+M N V+\frac{M N V}{\sqrt{T}}+M N V T .
$$

All terms can be made arbitrarily small choosing $T$ sufficiently large and $V$ small. This is obvious for all of them, except for the first one, where $c_{s_{1}}\left(s_{2}, \phi\right)$ appears in the definition of $\lambda$. However, substituting $\lambda$ we get

$$
c_{s_{1}}\left(s_{2}, \phi\right) \leq \frac{\sqrt{2 \epsilon M N V+c(\phi)+\operatorname{diam}\left(\mathbb{T}^{d}\right) c_{s_{1}}\left(s_{2}, \phi\right)}}{\sqrt{T}}+O(M N V T),
$$

which, in particular, implies that $c_{s_{1}}\left(s_{2}, \phi\right)$ is small it $\mathrm{T}$ is large.

It follows from the third statement of Lemma 1 that we can naturally define an operator $\hat{K}_{s, t}^{\omega}$ in the space $C\left(\mathbb{T}^{d}\right) / \mathbb{R}$ of continuous functions modulus constants. As a consequence of Lemma 7 we get the following corollary.

Corollary 2. With probability 1 the following properties hold.

(1) $\hat{K}_{s, t}^{\omega} \hat{\phi}(\cdot, t)$ form a Cauchy family as $t$ tend to infinity.

(2) The class of functions $\hat{\psi}^{\omega}(\cdot, s)=\lim _{t \rightarrow \infty} \hat{K}_{s, t}^{\omega} \hat{\phi}(\cdot, t)$ is independent of functions $\psi(x, t)$.

(3) $\hat{K}_{s, t}^{\omega} \hat{\psi}^{\omega}(\cdot, t)=\hat{\psi}^{\omega}(\cdot, s)$.

Proof: Fix $\delta>0$ and let $C$ be the constant and $\Sigma$ the set given by the second part of lemma 2. Choose $\alpha$ and $T$ such that lemma 7 holds for $\delta$ and $C$ as above. With probability 1 there exist an $\alpha$-weak interval of time $\left[t_{1}, t_{1}+T\right]$ with $t_{1}>s$. Moreover, we can assume that $\sigma^{t_{1}+T} \omega$ is in the set $\Sigma$. Indeed, the events of having a an interval with weak potentials is independent of the grow condition after the end of the interval.

Then if $t^{\prime}, t^{\prime \prime}$ are bigger than $t_{1}+T$ we claim that for arbitrary $\hat{\phi}_{1}, \hat{\phi}_{2}$

$$
\left|\hat{K}_{s, t^{\prime}}^{\omega} \psi-\hat{K}_{s, t^{\prime \prime}}^{\omega} \psi\right| \leq \delta
$$

Indeed, from the semigroup property we have

$$
\begin{aligned}
\hat{K}_{s, t}^{\omega} \hat{\phi}_{1} & =\hat{K}_{s, t_{1}}^{\omega} \circ \hat{K}_{t_{1}, t_{1}+T}^{\omega} \circ \hat{K}_{t_{1}+T, t^{\prime}}^{\omega} \hat{\phi}_{1}, \\
\hat{K}_{s, t^{\prime \prime}}^{\omega} \hat{\phi}_{2} & =\hat{K}_{s, t_{1}}^{\omega} \circ \hat{K}_{t_{1}, t_{1}+T}^{\omega} \circ \hat{K}_{t_{2}, t^{\prime \prime}}^{\omega} \hat{\phi}_{2} .
\end{aligned}
$$

Denote $\hat{\psi}_{1}=K_{t_{1}+T, t^{\prime}}^{\omega} \hat{\phi}_{1}$ and $\hat{\psi}_{2}=K_{t_{2}, t^{\prime \prime}}^{\omega} \hat{\phi}_{2}$. It follows from Lemma 7 that $\left\|\hat{K}_{t_{1}, t_{2}}^{\omega} \hat{\phi}_{1}-\hat{K}_{t_{1}, t_{2}}^{\omega} \hat{\phi}_{2}\right\| \leq 2 \delta$. Then the weak contraction property guarantees that the distances do not increase which proves (37) and 
hence the Cauchy property. The same argument proves the independence from the final condition.

Finally, for the proof of the last statement we have

$$
\begin{aligned}
\hat{\psi}^{\omega}\left(\cdot, s_{1}\right) & =\lim _{t \rightarrow \infty} \hat{K}_{s_{1}, t}^{\omega} \hat{\phi}=\lim _{t \rightarrow \infty} \hat{K}_{s_{1}, s_{2}}^{\omega} \hat{K}_{s_{2}, t}^{\omega} \psi \\
& =\hat{K}_{s_{1}, s_{2}}^{\omega} \lim _{t \rightarrow \infty} \hat{K}_{s_{2}, t}^{\omega} \psi=\hat{K}_{s_{1}, s_{2}}^{\omega} \hat{\phi}^{\omega}\left(x, s_{2}\right)
\end{aligned}
$$

Proof of Theorem 1: Fix $x_{0}$ a point in $T^{n}$, and define $\psi^{\omega}(x, s)$ as the unique function in the class of $\hat{\phi}^{\omega}(x, s)$ such that $\psi^{\omega}\left(x_{0}, s\right)=0$. Then there exist a $c(s)$ such that $\psi^{\omega}(x, 0)=K_{0, s}^{\omega} \psi^{\omega}(x, s)+c(s)$. Finally define $\phi^{\omega}(x, s)=\psi^{\omega}(x, s)-c(s)$, so clearly $\phi^{\omega}(x, 0)=K_{0, s}^{\omega} \psi^{\omega}(x, s)$, moreover for any $s<t$ we have

$$
K_{s, t}^{\omega} \phi^{\omega}(\cdot, t)=\phi^{\omega}(\cdot, s) .
$$

Indeed, there is a $c(s, t)$ such that

$$
K_{s, t}^{\omega} \phi^{\omega}(\cdot, t)=\phi^{\omega}(\cdot, s)+c(s, t)
$$

SO

$$
\begin{aligned}
K_{0, s}^{\omega} \circ K_{s, t}^{\omega} \phi^{\omega}(\cdot, t) & =K_{0, s}^{\omega} \phi^{\omega}(\cdot, s)+c(s, t), \\
K_{0, t}^{\omega} \phi^{\omega}(\cdot, t) & =K_{0, s}^{\omega} \phi^{\omega}(\cdot, s)+c(s, t), \\
\phi^{\omega}(\cdot, 0) & =\phi^{\omega}(\cdot, 0)+c(s, t)
\end{aligned}
$$

hence $c(s, t)=0$.

In the other hand $\psi^{\omega}(x, s)$ is another solution of the Hamilton Jacobi equation, it is necessarily satisfies $(28)$, so it has to be $\phi^{\omega}(x, s)$.

\section{Convergence of viscosity SOlutions}

In this section we prove Theorems 2 - 4 . As above the value of the parameter $b$ is fixed and omitted. As in the previous section, we give proofs in the case of negative viscosity $\nu$ and use notation $\psi_{\epsilon}^{\omega}$ for the unique global solution constructed in Theorem 1 , where $\epsilon=-\nu>0$. We assume that $\psi_{\epsilon}^{\omega}\left(x_{0}, 0\right)=0$ for a fixed point $x_{0} \in \mathbb{T}^{d}$. We shall also denote by $K_{s_{1}, s_{2}}^{\omega, \epsilon}$ and $K_{s_{1}, s_{2}}^{\omega, 0}$ the Lax operators in the viscous and inviscid cases respectively, where $K_{s_{1}, s_{2}}^{\omega, \epsilon}$ is given by (27) and

$$
K_{s_{1}, s_{2}}^{\omega, 0} \phi(x)=\sup _{\gamma}\left[\phi\left(\gamma\left(s_{2}\right)\right)-A_{s_{1}, s_{2}}^{\omega}(\gamma)\right] .
$$


The supremum in the formula above is taken over all absolutely continuous curves $\gamma:\left[s_{1}, s_{2}\right] \rightarrow \mathbb{T}^{d}, \gamma\left(s_{1}\right)=x$ and

$$
\begin{aligned}
& A_{s_{1}, s_{2}}^{\omega}(\gamma)=\int_{s_{1}}^{s_{2}} L^{\omega, 0}(\gamma(\tau), \dot{\gamma}(\tau), \tau)=\sum_{i=1}^{N} F_{i}(x)\left(W_{i}\left(s_{1}\right)-W_{i}\left(s_{2}\right)\right)+ \\
& \int_{s_{1}}^{s_{2}}\left[\frac{1}{2}|\dot{\gamma}(\tau)|^{2}+\sum_{i=1}^{N} \nabla F_{i}\left((\gamma(\tau)) \cdot \dot{\gamma}(\tau)\left(W_{i}(\tau)-W_{i}(t)\right)\right] d \tau .\right.
\end{aligned}
$$

Finally, we shall denote by $\psi_{0}^{\omega}$ the unique (see [14]) global solution in the inviscid case which also satisfies the condition $\psi_{0}^{\omega}\left(x_{0}, 0\right)=0$. In all the proofs below it is assumed that $\omega$ belongs to a subset of full measure for which all Wiener processes $W_{i}(t), 1 \leq i \leq N$ are continuous and uniqueness holds for global solutions of the Hamilton-Jacobi equation.

Lemma 9. Let $\epsilon_{k}$ be a sequence such that $\epsilon_{k} \rightarrow 0$ as $k \rightarrow \infty$. Assume that for some $s_{1}<s_{2}$ solutions $\psi_{\epsilon_{k}}^{\omega}\left(x, s_{1}\right)$ and $\psi_{\epsilon_{k}}^{\omega}\left(x, s_{2}\right)$ uniformly converge to $\psi_{1}(x)$ and $\psi_{2}(x)$ respectively in the limit $k \rightarrow \infty$. Then, $\psi_{1}(x)=K_{s_{1}, s_{2}}^{\omega, 0} \psi_{2}(x)$.

Proof: We first prove that $\psi_{1}(x) \geq K_{s_{1}, s_{2}}^{\omega, 0} \psi_{2}(x)$. Indeed, if $\psi_{1}(x)<$ $K_{s_{1}, s_{2}}^{\omega, 0} \psi_{2}(x)$, then there exist $\delta>0$ and a $C^{1}$-smooth curve $\gamma$ such that $\gamma\left(s_{1}\right)=x$ and $\psi_{1}(x)<\psi_{2}\left(\gamma\left(s_{2}\right)\right)-A_{s_{1}, s_{2}}^{\omega}(\gamma)-\delta$. Taking a control $u(\tau)=\dot{\gamma}(\tau)$ and $k$ so large that $\left\|\psi_{\epsilon_{k}}^{\omega}\left(x, s_{1}\right)-\psi_{1}(x)\right\| \leq \delta / 6, \| \psi_{\epsilon_{k}}^{\omega}\left(x, s_{2}\right)-$ $\psi_{2}(x) \| \leq \delta / 6$ and

$$
\begin{aligned}
& \|\left(\psi_{2}\left(\gamma\left(s_{2}\right)\right)-A_{s_{1}, s_{2}}^{\omega}(\gamma)\right) \\
& \quad-E\left[\psi_{\epsilon_{k}}^{\omega}\left(x^{u}\left(s_{2}\right), s_{2}\right)-\int_{s_{1}}^{s_{2}} L\left(x^{u}(\tau), u(\tau), \tau\right) d \tau\right] \| \leq \frac{\delta}{6}
\end{aligned}
$$

we have $\psi_{\epsilon_{k}}^{\omega}\left(x, s_{1}\right)<K_{s_{1}, s_{2}}^{\omega, \epsilon_{k}} \psi_{\epsilon_{k}}^{\omega}\left(x, s_{2}\right)-\delta / 2$ which is a contradiction since $\psi_{\epsilon_{k}}^{\omega}$ is a solution to the viscous Hamilton-Jacobi equation. On the other hand, using Lemma 6 it is easy to show that for any $\delta>$ 0 there exists an absolutely continuous curve $\gamma$ such that $\psi_{1}(x)$ and $\left(\psi_{2}\left(\gamma\left(s_{2}\right)\right)-A_{s_{1}, s_{2}}^{\omega}(\gamma)\right)$ are $\delta$-close. This implies $\psi_{1}(x) \leq K_{s_{1}, s_{2}}^{\omega, 0} \psi_{2}(x)$

Proof of Theorem 2: For all $t$, the family $\psi_{\epsilon}^{\omega}(x, t)$ is a compact family in $C\left(\mathbb{T}^{d}\right)$. Choose a sequence $\epsilon_{k} \rightarrow 0$ such that for all integer $t=l$ solutions $\psi_{\epsilon}^{\omega}(x, l)$ uniformly converge to $\psi(x, l)$. Using Lemma 9 we conclude that $\psi(x, l)$ generates a global solution to the inviscid Hamilton-Jacobi equation. Since $\psi\left(x_{0}, 0\right)=0$, it follows from uniqueness that $\psi(x, l)=\psi_{0}^{\omega}(x, l)$. This implies that $\psi_{\epsilon}^{\omega}(x, l)$ uniformly converges to $\psi_{0}^{\omega}(x, l)$ as $\epsilon \rightarrow 0$. Applying Lemma 9 once again we get uniform convergence for all $t \in \mathbb{R}$. 
Recall that a plane of support $\xi$ at a point $x$ for a concave function $f$ is an afine map such that $\xi_{n}(x)=f_{n}(x)$ and $\xi_{n}(y) \geq f_{n}(y)$.

Lemma 10. If $f_{n} \rightarrow f_{0}$ is a uniformly convergent sequence of concave functions and $\xi_{n}$ is a plane of support of $f_{n}$ at the point $x$, further assume that $\xi_{n}$ converges punctually $\xi_{0}$, as $n$ tends to infinity. Then $\xi_{0}$ is a plane of support of $f_{0}$ at $x$.

Proof: Assume otherwise, as $\xi_{n}(x)=f_{n}(x)$ then as $n$ tends to infinity we conclude that $\xi_{0}(x)=f_{0}(x)$, so suppose that there exists $y$ such that $\xi_{0}(y)<f_{0}(y)$. From the uniform convergence of $f_{n}$ and the punctual convergence of $\xi_{n}$ we conclude that if $n$ is big enough then $\xi_{n}(y)<f_{n}(y)$ contradiction with the fact that $\xi_{n}$ is a plane of support of $f_{n}$.

Proof of Theorem 3: Recall that at points of differentiability of concave function there is only one support plane given by the differential, see for example [17].

From theorem 2 and lemma 6 we may assume that $\phi_{\epsilon}^{\omega}(x, t)-C_{2}(\omega) x^{2}$ is a concave function that converges uniformly to $\phi_{0}^{\omega}(x, t)-C_{2}(\omega) x^{2}$ also a concave function. Moreover we are assuming that $\phi_{0}^{\omega}(x, t)$ is differentiable at $x_{0}$, so $\phi_{0}^{\omega}(x, t)-C_{2}(\omega) x^{2}$ is also differentiable. Let $\phi_{\epsilon_{k}}^{\omega}$ any subsequence, the differentials are bounded, so there is a further subsequence $\phi_{\epsilon_{k_{j}}}^{\omega}$ with convergent differentials. This implies that the plane of support converge given by the differentials of $\phi_{\epsilon_{k_{j}}}^{\omega}(x, t)-C_{2}(\omega) x^{2}$ converge.

From the previous lemma 10 we conclude that convergence is to the to the unique plane of support of $\phi_{0}^{\omega}(x, t)-C_{2}(\omega) x^{2}$. This implies the convergence of the differential of $\phi_{\epsilon}^{\omega}(x, t)$ at $x_{0}$.

Proof of Theorem 4: The proof of the first statement is the same as the proof of the similar statements in [6] and [14]. It immediately follows from the convergence of solutions to the Cauchy problems and their gradients in the limit as $T_{0} \rightarrow-\infty$.

To prove the second statement notice that

$$
m_{\nu}=\int_{\Omega} \delta\left(\hat{\psi}_{\nu}^{\omega}(x, 0)\right) P(d \omega), \mu_{\nu}=\int_{\Omega} \delta\left(\nabla \psi_{\nu}^{\omega}(x, 0)\right) P(d \omega)
$$

and

$$
m=\int_{\Omega} \delta\left(\hat{\psi}_{-}^{\omega}(x, 0)\right) P(d \omega), \mu=\int_{\Omega} \delta\left(\nabla \psi_{-}^{\omega}(x, 0)\right) P(d \omega) .
$$

It follows from Theorem 2 that $\hat{\psi}_{\nu}^{\omega}(x, 0)$ converge to $\hat{\psi}_{-}^{\omega}(x, 0)$ in $C\left(\mathbb{T}^{d}\right) / \mathbb{R}$. This implies weak convergence $m_{\nu} \rightarrow m$ as $\nu \rightarrow 0$. Finally, notice that Theorem 3 and Lemma 6 give convergence $\nabla \psi_{\nu}^{\omega}(x, 0) \rightarrow \nabla \psi_{-}^{\omega}(x, 0)$ in 
$L^{p}\left(\mathbb{T}^{d}\right)$ for any $p \geq 1$, which implies weak convergence $\mu_{\nu} \rightarrow \mu$ in the space of probability distributions on $L^{p}\left(\mathbb{T}^{d}\right)$.

\section{Conclusions}

1. In this paper we present a simple approach which allows to construct stationary distributions for random forced viscous HamiltonJacobi and Burgers equations and to prove convergence of these distributions in the limit of vanishing viscosity. The key fact is the uniqueness of global solutions which satisfy uniform Lipschitz and semiconvexity properties. We restrict our attention to the Lagrangian

$$
L(x, v, t)=L_{0}(x, v)-F(x, t)
$$

where $L_{0}(x, v)=v^{2} / 2$. However, the results hold in much more general situations. For example, it is straightforward to replace $v^{2} / 2$ with a metric $a(x) v^{2}$. In fact, we strongly believe that most of the results of this paper can be extended to a wide class of Lagrangians $L_{0}$ on compact Riemannian manifolds. One can also consider other types of forcing terms, for example kicked force. In this situation the force is applied instantly in periodic moments of time (see [14]). One can also consider non-random "generic" time-dependent Lagrangian systems. The notion of stationary distribution is not applicable in this case but uniqueness of global solutions still holds. We shall discuss all generalizations mentioned above in a forthcoming publication.

2. The method used in this paper uses the "fluctuational mechanism" for the uniqueness of a global solution and convergence of the solutions to the Cauchy problems in the limit $T_{0} \rightarrow \pm \infty$ (see Theorem 1 ). The nature of the fluctuational mechanism is very general but it guarantees only a very slow rate of convergence. At the same time it is clear that convergence in Theorem 1 should be exponentially fast in $\left|T_{0}\right|$. In the case of viscous Hamilton-Jacobi and Burgers equations $\left(L_{0}(x, v)=v^{2} / 2\right)$ the exponential convergence was proven by Sinai ([18]) using the Hopf-Cole transformation and Markov mechanism of contraction. It is very interesting to extend this result for the case of general Lagrangians. There are strong reasons to believe that the exponent for this exponential rate does not vanish in the limit of vanishing viscosity, and the limiting value is strongly related to the hyperbolic properties of minimizing orbits for a Lagrangian flow. 


\section{REFERENCES}

[1] E. Balkovsky, G. Falkovich, I. Kolokolov, and V. Lebedev, Intermittency of Burgers turbulence, Phys. Rev. Lett., 78 (1997), 1452-1455.

[2] J. Bec, U. Frisch and K. Khanin, Kicked Burgers Turbulence, J. Fluid Mech., 416 (2000), 239-267.

[3] S. Boldyrev, Velocity-difference probability density for Burgers turbulence, Phys. Rev. E, 55 (1997), 6907-6910.

[4] J. P. Bouchaud, M. Mezard and G. Parisi, Scaling and intermittency in Burgers turbulence, Phys. Rev. E, 52 (1995), 3656-3674.

[5] A. Chekhlov and V. Yakhot, Kolmogorov Turbulence in a random force-driven Burgers equation Phys. Rev E, 51 (1995), R2739-R2749.

[6] W. E, K. Khanin, A. Mazel and Ya. Sinai, Invariant measures for Burgers equation with stochastic forcing, Annals of Math., 151 (2000), 877-960.

[7] Lawrence C. Evans Partial Differential Equations, Graduate Studies in Mathematics vol 19, American Mathematical Society 1998.

[8] A. Fathi, Théorème KAM faible et Théorie de Mather sur les systems Lagrangiens, C.R. Acad. Sci. Paris, t. 324, Série I (1997), 1043-1046.

[9] A. Fathi, Weak KAM Theorem in Lagrangian Dynamics, Preprint 2001 (to be published by the Cambridge University Press).

[10] Wendell H. Fleming, H. Mete Soner Controlled Markov Processes and Viscosity solutions Applications of Mathematics 25 Springer Verlag 1993.

[11] U. Frisch, J. Bec and B. Villone, Singularities and the distribution of density in the Burgers/adhension model, Physica D, (to appear, 2000).

[12] T. Gotoh and R. H. Kraichnan, Burgers turbulence with large scale forcing, Phys. Fluids A, 10 (1998), 2859-2866.

[13] V. Gurarie and A. Migdal, Instantons in Burgers equations, Phys. Rev. E, 54 (1996), 4908-4914.

[14] R. Iturriaga and K. Khanin, Burgers Turbulence and Random Lagrangian Systems preprint (2001).

[15] P. L. Lions, Generalized Solutions of Hamilton-Jacobi Equations, Research Notes in Math., 69, Pitman Advanced Publishing Program, Boston, 1982.

[16] A. Polyakov, Turbulence without pressure, Phys. Rev E, 52 (1995), 6183-6188.

[17] R.T. Rockafellar. Convex Analysis Princeton Landmarks in Math. Princeton. Princeton University Press (1997)

[18] Ya. Sinai, Two results concerning asymptotic behavior of solutions of the Burgers equation with force, J. Stat. Phys., 64 (1992), 1-12.

D. Gomes :

Instituto Superior TÉCNICO

DeP. DE MATEMÁtica

Av. Rovisco Pais

Lisboa, Portugal

E-mail address: dgomes@math.ist.utl.pt 
R. ITURRIAGA:

Centro de Investigacion en Matematica,

Apartado Postal 402, Guanajuato Gto., 36000 Mexico

E-mail address: renato@cimat.mx

K. KHANIN:

Isaac Newton Institute for Mathematical Sciences,

20 Clarkson Road, Cambridge CB3 0EH, UK

Heriot-Watt University, Edinburgh, UK

Landau Institute, Moscow, Russia

E-mail address: kk262@newton.cam.ac.uk

P. PADILLA:

IIMAS, Universidad Nacional Autonoma de Mexico,

Circuito Escolar, CD. Universitaria, México D.F. 04510, México

E-mail address: pablo@uxmym1.iimas.unam.mx 